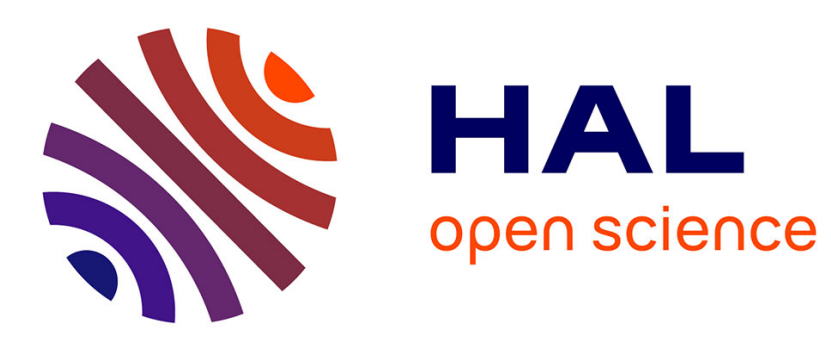

\title{
Digital implementation of Hodgkin-Huxley neuron model for neurological diseases studies
}

Timothée Levi, Farad Khoyratee, Sylvain Saïghi, Yoshiho Ikeuchi

\section{To cite this version:}

Timothée Levi, Farad Khoyratee, Sylvain Saïghi, Yoshiho Ikeuchi. Digital implementation of HodgkinHuxley neuron model for neurological diseases studies. 22th International Symposium on Artificial Life and Robotics, Jan 2017, Beppu, Japan. hal-01567496

\section{HAL Id: hal-01567496 \\ https://hal.science/hal-01567496}

Submitted on 25 Jul 2017

HAL is a multi-disciplinary open access archive for the deposit and dissemination of scientific research documents, whether they are published or not. The documents may come from teaching and research institutions in France or abroad, or from public or private research centers.
L'archive ouverte pluridisciplinaire HAL, est destinée au dépôt et à la diffusion de documents scientifiques de niveau recherche, publiés ou non, émanant des établissements d'enseignement et de recherche français ou étrangers, des laboratoires publics ou privés. 


\title{
Digital implementation of Hodgkin-Huxley neuron model for neurological diseases studies
}

\author{
Timothée Levi ${ }^{1}$, Farad Khoyratee ${ }^{1}$, Sylvain Saïghi ${ }^{1}$, Yoshiho Ikeuchi ${ }^{2}$ \\ ${ }^{1}$ IMS Lab., University of Bordeaux, France \\ ${ }^{2}$ Institute of Industrial Science, The University of Tokyo, Japan \\ (Tel: 33-5-4000-3118, Fax: 33-5-5637-1545) \\ ${ }^{1}$ timothee.levi@u-bordeaux.fr
}

\begin{abstract}
Neurological disorders affect millions of people which influence their cognitive and/or motor capabilities. The realization of a prosthesis must consider the biological activity of the cells and the connection between machine and biological cells. Biomimetic neural network is one solution in front of neurological diseases. The neuron replacement should be processed by reproducing the timing and the shape of the spike. Several mathematical equations which model neural activities exist. The most biologically plausible one is the Hodgkin-Huxley $(\mathrm{HH})$ model. The connection between electrical devices and living cells require a tunable real-time system. The FPGA (Field Programmable Gate Array) is a nice component including flexibility, speed and stability. Here we propose an implementation of HH neurons in FPGA serving as a presage for a modulating network opening a large scale of possibilities like damage cells replacement and the study of the effect of the cells disease on the neural network.
\end{abstract}

Keywords: Silicon neuron, Hodgkin-Huxley, FPGA, neurological diseases

\section{INTRODUCTION}

Millions of people worldwide are affected by neurological disorders which could cause variety of symptoms including paralysis and dementia. Such a number is likely to increase in the next years and current assistive technology is still limited. Since last decades Brain-Machine Interfaces (BMIs) and generally neuroprosthesis [1-4] have been object of extensive research and may represent a valid treatment for such disabilities. The development of these devices has and will hopefully have a profound social impact on the quality of life. The realization of such prostheses implies that we know how to interact with neuronal cell assemblies, taking into account the intrinsic spontaneous activity of neuronal networks and understanding how to drive them into a desired state or to produce a specific behavior. The long-term goal of replacing damaged brain areas with artificial devices also requires the development of neuronal network models. They will fit with the recorded electrophysiological patterns and will produce in their turn the correct stimulation patterns for the brain so as to recover the desired function. The hardware set-up that will be used to interface the biological component is a Spiking Neural Network (SNN) system [5-8]. SNN implements biologically realistic neural network models, spanning from the electrophysiological properties of one single neuron up to network plasticity rules. Biomimetic neural network is one solution in front of neurological diseases. The neuron replacement should be processed by reproducing the timing and the shape of the spike.

In the human brain, types of neurons are radically different. Complexities of neuronal cells are abstracted by a wide variety of methodology to help the understanding of the different aspects of neural networks' development, function, or learning. The neuronal model is a mathematical description of the electrophysiological properties of neuronal cells, or neurons. It tries to accurately describe and predict their biological processes.

In order to provide a SNN, the first step is the choice of the neuromimetic mathematical model. Indeed, the mathematical model, based on differential equation, can reproduce a behavior more or less close to biological cell. In order to choose our model, we are looking two criteria: the variety of firing rate dynamics that can be reproduced and the shape of action potential for modelling neurological disease. Regarding those criteria, we can compare all models like threshold-models: the Leaky Integrate and Fire (LIF) [9] or the Izhikevich model (IZH) [10] or like complete model: the Hodgkin-Huxley model (HH) [11]. The HH model can reproduce all kind of neurons with a good accuracy in terms of shape of spike or complex firing activities compared to threshold models. The drawback is its computation cost but our objective is to simplify this model for a few resource implementation in to digital board.

Various software solutions are currently available for simulating neuron models. Less conventional than softwarebased systems, hardware based solutions are also provided 
which generally combine digital and analog forms of computation. Analog implementations are fast and efficient however they are inflexible, sensitive to variations and require a long development time. As in many other fields of microelectronics, a mixed implementation offers both the advantages and disadvantages of both solutions: analog circuits have a higher integration density, and digital platforms have better programmability. Recently, as a midpoint in the design space, FPGAs have been used to build spiking neuronal networks. Digital FPGA implementations offer a significant speedup over software designs, as well as size, weight, and power efficiency. Compared to analog VLSI, digital FPGAs designs are stable and flexible in design alterations. Previous works have already implemented neuronal networks on FPGA [12-14]. However, those designs have been realized for computation purposes without taking into account biological real time. Few FPGA implementation are used for hybrid experiments [15-17] but they used threshold neuron model. The implementation in FPGA of HH model are rare [18], [19]. These works are used for neural network simulation (accelerated-time) and they are not optimized in terms of surface and number of neurons.

In this paper, we present the implementation in FPGA platform of $\mathrm{HH}$ neurons dedicated to the simulation of neurological diseases.

\section{HODGKIN-HUXLEY FORMALISM}

\subsection{Equations}

The first biologically relevant mathematical neuron model was proposed in 1952 by Hodgkin and Huxley (HH). Their four-dimensional set of equations describes the ionic conductance's dynamics of the giant axon, which are the starting point for detailed neuron models which account for numerous ion channels, different types of synapses, and the specific spatial geometry of an individual cell. Such class of models, conductance based models, successfully describe and reproduce the neuronal activity. Moreover the parameters of the HH model have biophysics meaning. Thus, in case of study of neurological diseases, we can modify the original model to easily include the cell degeneration.

The main advantage of this formalism is that it relies on biophysically realistic parameters and describes individual ionic and synaptic conductances for each neuron in accordance with the dynamics of ionic channels. Electrical activity of a neuron is the consequence of the diffusion of different ionic species through its membrane. The $\mathrm{HH}$ formalism provides a set of equations and an equivalent electrical circuit (Figure 1), which describes the conductance interplay underlying the genesis of action potentials.

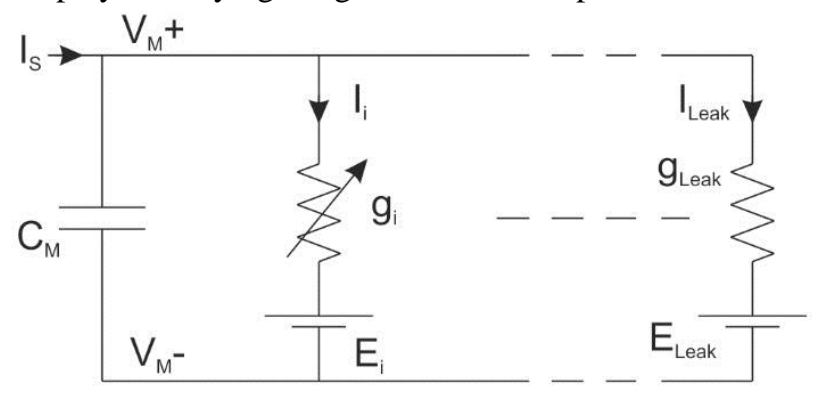

Fig. 1. Equivalent electrical circuit of a neuron.

The current flowing across the membrane is integrated on the membrane capacitance, according to expression (1):

$$
\mathrm{C}_{\mathrm{M}} \frac{\mathrm{dV}_{\mathrm{M}}}{\mathrm{dt}}=-\sum_{\mathrm{i}} \mathrm{I}_{\mathrm{i}}+\mathrm{I}_{\text {Leak }}+\mathrm{I}_{\mathrm{S}}
$$

Where VM is the membrane potential, $\mathrm{CM}$ is the membrane capacitance, Ii denotes the individual ionic currents of the model, ILeak the leakage current, and IS is a stimulation or a synaptic current. Ii is the current for a given type of channel, and its associated equation is:

$\mathrm{I}_{\mathrm{i}}=\mathrm{g}_{\mathrm{i}} \cdot \mathrm{m}^{\mathrm{p}} \cdot \mathrm{h}^{\mathrm{q}} \cdot\left(\mathrm{V}_{\mathrm{M}}-\mathrm{E}_{\mathrm{i}}\right)$

Where gi is the maximum conductance; $\mathrm{m}$ and $\mathrm{h}$ are gating variables for activation and inactivation, respectively, representing the fraction of open gates available at any given time and voltage. Ei is the ion-specific reversal potential and $\mathrm{p}$ and $\mathrm{q}$ are integers.

$\mathrm{C}_{\mathrm{M}} \frac{\mathrm{dV}_{\mathrm{M}}}{\mathrm{dt}}=-\sum_{\mathrm{i}} \mathrm{I}_{\mathrm{i}}+\mathrm{I}_{\text {Leak }}+\mathrm{I}_{\mathrm{S}}$

According to Hodgkin and Huxley [11], the values of activation and inactivation's parameters are updated by equation (4).

$$
\frac{d x}{d t}=\frac{x_{\infty}(V)-x}{\tau_{x}(V)}
$$

Where $\mathrm{x} \in\{\mathrm{m}, \mathrm{n}, \mathrm{h}\}$ and $\tau_{\mathrm{x}}(\mathrm{V})$ and $\mathrm{x}(\mathrm{V})$ are obtained by (5) and (6).

$$
\begin{aligned}
& \tau_{x}(V)=\frac{1}{\alpha_{x}(V)+\beta_{x}(V)} \\
& x_{\infty}(V)=\frac{\alpha_{x}(V)}{\alpha_{x}(V)+\beta_{x}(V)}
\end{aligned}
$$

Where $\alpha$ and $\beta$ are exponential-based equations defined in [11]. 


\subsection{Implementation}

The connection between electrical devices and living cells requires a real-time system. In addition, studying neurodegenerative troubles requires a tunable appliance. The FPGA (Field Programmable Gate Array) is the best component including flexibility, speed and stability. Here we propose biomimetic neurons designed by FPGA as a central processing unit and DAC (Digital to Analog Converter) as the spike generator. The system is integrating a configurable main unit managing the computation neuron core, the sampling and the communication with the DAC.

The implementation has been set on the "Genesys 2" board which contains a Kintex 7 FPGA "xc7k325tffg900". The signal has been output using the DA4 Pmod from Digilent which has an AD5628 Digital to Analog Converter (DAC) of 12 bits. The frequency of the system has been assigned at 200MHz. A length of 32bits (16 decimals) has been used as data for the operations. A pipelined architecture is used (see fig. 2) using the Euler method to solve differential equation and Taylor series for exponentials calculations. A $\Delta t$ sampling time of $50 \mu$ s is used for the Euler method.

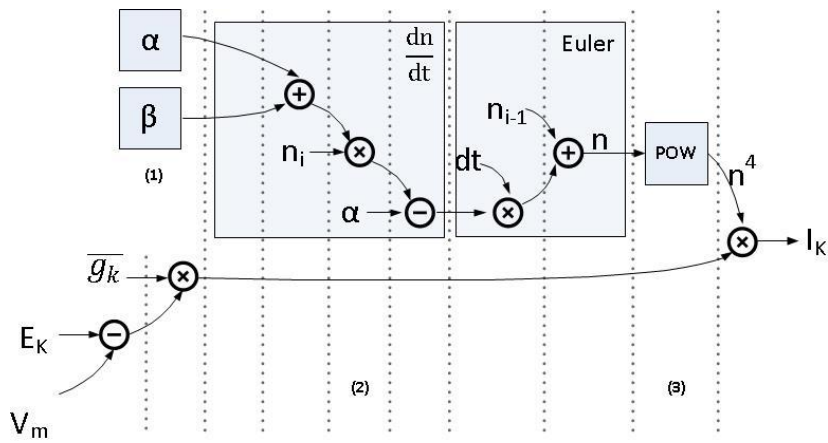

Fig. 2. Pipeline architecture of an ionic current which take 400 cycles (1) Alpha and Beta has to be calculated to continue the operation (2) According to the kintex 7 DSP, input and output of the multiplier need to be pipelined and 9 cycles is used to perform the operation. (3) The power module has a number of cycles depending of operations order

The result show limited resources taken into the FPGA for the calculation of 25 neurons (table 1).

Table 1. Device resources using xc7k325tffg900 kintex 7 FPGA for $25 \mathrm{HH}$ neurons

\begin{tabular}{|c|c|c|}
\hline & Used & Utilization (\%) \\
\hline LUT & 15481 & 7.6 \\
\hline LUTRAM & 72 & 0.11 \\
\hline FF & 13040 & 3.2 \\
\hline DSP & 132 & 15.71 \\
\hline
\end{tabular}

\section{RESULTS}

\subsection{Comparaison Matlab FPGA}

The results show similar dynamics for ionic currents comparing the simulation on Matlab and the output of the DAC (fig. 3).

(A)

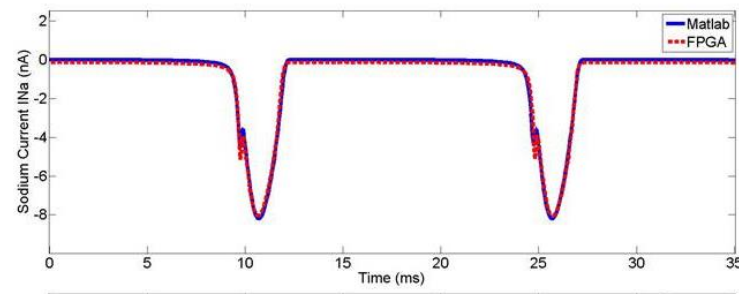

(B)

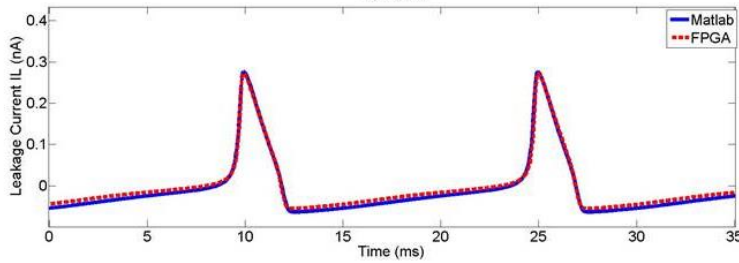

(C)

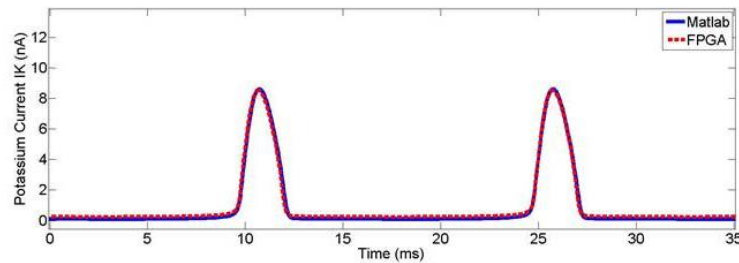

Fig. 3. Comparison of the results from the FPGA and from Matlab (A) Sodium current (B) Potassium current (C) Leakage current

Also, we notice that membrane potential from Matlab and from FPGA are similar in terms of amplitude and frequency (fig 4).

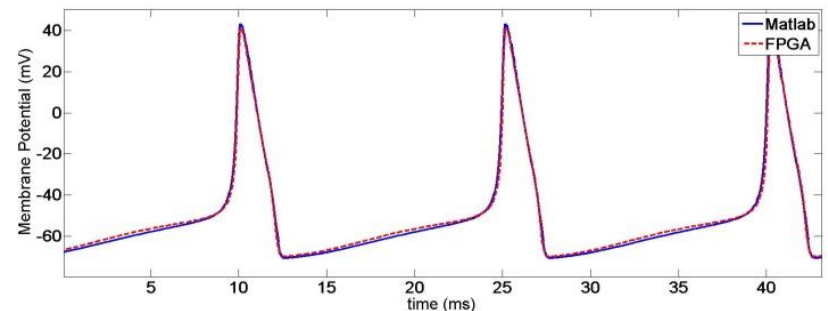

Fig. 4. Comparison of the action potential behavior from the FPGA and from Matlab

These results shows that our implementation in FPGA is valid and that our computation methods (number of bits, Euler method) are valid.

\subsection{Neurological disease applications}

This HH implementation is used to model neurological disease. From recordings of biological action potential data, our goal is to model the differences between healthy and disease neurons. 
Thanks to collaboration with neuroscientists [20], we are working on Tuberous Sclerosis disease. When neuroscientists model Tuberous Sclerosis in 'in vitro' neuron culture, they observe bigger neuron soma than the control cells. This behavior can be reproduced by $\mathrm{HH}$ model by tuning the parameters [21].

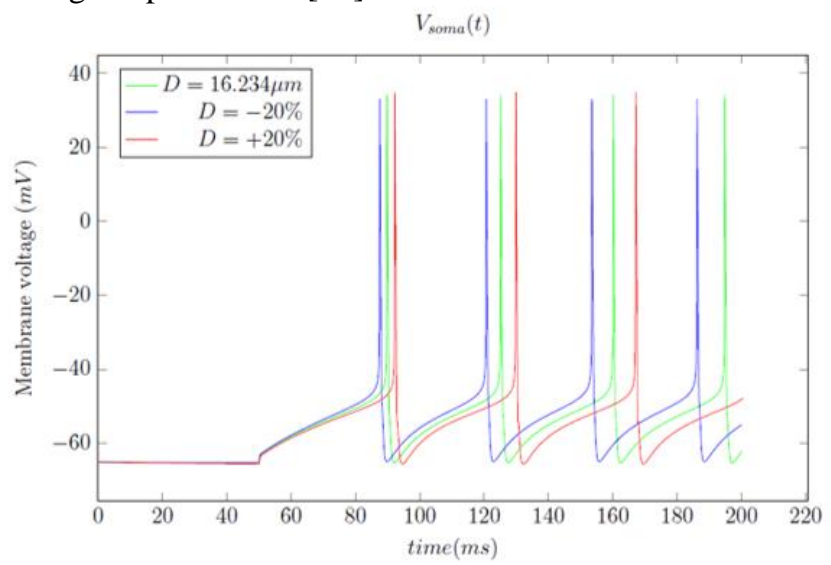

Fig. 5. Action potential behaviors following the diameter of neuron soma.

Fig. 5 describes that following the size of the neuron soma, amplitude and frequency of action potential change. When the soma size increases, amplitude is higher and frequency is lower.

\section{CONCLUSION}

We presented, in this paper, an implementation of single compartment $\mathrm{HH}$ model in FPGA. This system works in real-time and can perform bio-hybrid experiments. We validate our implementation by comparison with software. In the next future, we will implement compartmental $\mathrm{HH}$ model, synapses and long-term plasticity to this neural network.

The final goal of the studies is to model neurological disease and implement a 'disease' neuron by $\mathrm{HH}$ parameters and then study the influence and the behavior of these neurons integrated into a neural network.

\section{ACKNOWLEDGMENTS}

The research leading to these results has received funding from CNRS PEPS2015 "Neuroprotest".

\section{REFERENCES}

[1] Nicolelis, M. A.L., and Lebedev, M. A. (2009), Principles of neural ensemble physiology underlying the operation of brain-machine interfaces, Nature Reviews Neuroscience, 10, 530-540

[2] Hochberg, L.R., Serruya, M.D., Friehs, G.M., Mukand, J.A., Saleh, M., Caplan, A.H., Branner, A., Chen, D., Penn,
R.D., and Donoghue, J.P. (2006), Neuronal ensemble control of prosthetic devices by a human with tetraplegia, Nature, $442,164-171$

[3] Hochberg, L.R., Bacher, D., Jarosiewicz, B., Masse, N.Y., Simeral, J.D., Vogel, J., Haddadin, S., Liu, J., Cash, S.S., Van Der Smagt, P., and Donoghue, J.P. (2012), Reach and grasp by people with tetraplegia using a neurally controlled robotic arm, Nature Methods, 485, 372-375

[4] Bonifazi P, Difato F, Massobrio P, Breschi GL, Pasquale V, Levi T, Goldin M, Bornat Y, Tedesco M, Bisio M, Kanner S, Galron R, Tessadori J, Taverna S and Chiappalone M (2013), In vitro large-scale experimental and theoretical studies for the realization of bi-directional brainprostheses. Frontiers in Neural Circuits, 7:40

[5] Levi T, Lewis N, Tomas J, Saighi S, Renaud S, Bornat Y, Alvado L (2008), Neuromimetic Integrated Circuits, VLSI Circuits for Biomedical Applications, Iniewski Editor

[6] Mahowald M., R. Douglas R. (1991), A Silicon neuron, Nature, 1991: 515-518

[7] Indiveri G. et al. (2001), Neuromorphic silicon neuron circuits, Frontiers in Neuroscience, 5:73

[8] Levi T, Lewis N, Tomas J, Fouillat P (2008), IP-based methodology for analog design flow: application on neuromorphic engineering, NEWCAS-TAISA Conference, 343-346

[9] Indiveri G (2007), Synaptic plasticity and spike-based computation in VLSI networks of integrate-and-fire neurons. Neural Information Processing, Letters and Reviews 11:135146

[10] Izhikevich, E.M. (2003), Simple model of spiking neurons, IEEE Transactions on Neural Networks, vol.14, no.6, 1569-1572

[11] Hodgkin AL, Huxley AF (1952), A quantitative description of membrane current and its applications to conduction and excitation in nerve, The Journal of Physiology, 117:500-544

[12] Cassidy, A., Andreou, A.G (2008), Dynamical digital silicon neurons, IEEE Biomedical Circuits and Systems Conference, pp.289-292, 20-22

[13] Cassidy, A., Andreou, A.G., Georgiou, J. (2011), Design of a one million neuron single FPGA neuromorphic system for real-time multimodal scene analysis, 45th Annual Conference CISS, 1-6

[14] Basham, E.J, Parent D.W (2012), Compact digital implementation of a quadratic integrate-and-fire neuron, 34th Annual Conference of the IEEE EMBS, 3543-8

[15] Ambroise M, Levi T, Joucla S, Yvert B, Saighi S. (2013), Real-time biomimetic Central Pattern Generators into FPGA for hybrid experiments, Frontiers in Neuroscience, 7: 215

[16] Grassia F., Levi T., Kohno T., Saighi S. (2014), Silicon neuron: digital hardware implementation of the quartic model, Journal of Artificial Life and Robotics, 19:215-219

[17] Joucla S, Ambroise M, Levi T, Lafon T, Chauvet P, Saïghi S, Bornat Y, Lewis N, Renaud S and Yvert B (2016), Generation of Locomotor-Like Activity in the Isolated Rat Spinal Cord Using Intraspinal Electrical Microstimulation Driven by a Digital Neuromorphic CPG. Frontiers in 
Neurosciences, 10:67

[18] Bonabi Y, Asgharian H., Safari S. and Ahmadabadi M. (2014), FPGA implementation of a biological neural network based on the Hodgkin-Huxley neuron model, Frontiers in Neuroscience, 8:379

[19] Lu M, Wang J-L, Wen J, Dong X-W (2016), Implementation of Hodgkin-Huxlet neuron model in FPGAs, Asia-Pacific International Symposium on Electromagnetic Compatibility, 1:1115-1117

[20] Mejia L, Litterman N, Ikeuchi Y, De la Torre-Ubieta L, Bennett E, Zhang C, Harper W, Bonni A (2013), A Novel Hap1-Tsc1 Interaction Regulates Neuronal mTORC1 Signaling and Morphogenesis in the Brain, Journal of Neuroscience, 33:46, 18015-21

[21] Davison A, Feng J, Brown D (2000), A reduced compartmental model of the mitral cell for use in network models of the olfactory bulb, Brain Research Bulletin, 51:5, 393-399 